\title{
Interactive comment on "Archival of the water stable isotope signal in East Antarctic ice cores" by Mathieu Casado et al.
}

\section{Anonymous Referee \#1}

Received and published: 16 December 2016

It's critical to study the formation processes of the ice core records for a sound interpretation, especially for those ice cores recovered from the low accumulation sites of the East Antarctica, where the longest ice core record is found. This paper presents observations on the isotopic composition of the vapor, the precipitation, the surface and the buried snow samples collected from the inland of the East Antarctic Plateau, with purpose to understand the post-depositional processes affecting the variability of the isotopic composition in the buried snow. In general, the dataset is invaluable, especially from the brutal remote sites of the East Antarctica, and the study is quite comprehensive. However, the dataset is too limited to reach robust conclusions. For instance, the authors suggested enriched (depleted) heavy isotopes in the vapour (snow) during frost deposition events, but only a single frost event was sampled. There exists spatial variation of the stable isotopic composition, as also indicated by the authors, but it's 
hard to qualify its effects on the temporal change of the stable isotopic composition of the surface and the buried snow. New precipitation events, and possible snow drifting as well, during the observation further complicate the situation. The authors identified a common $20 \mathrm{~cm}$ cycle. Given the significantly different accumulation and sampling resolution, the common $20 \mathrm{~cm}$ cycle may have a quite different meaning. For example, at $\mathrm{S} 2$ with accumulation of $6.0 \mathrm{~cm}$ snow equivalent and finest resolution of $3 \mathrm{~cm}$, the $20 \mathrm{~cm}$ cycle may suggest multi-year variation. To the contrary, at South Pole with accumulation of $19.7 \mathrm{~cm}$ snow equivalent and finest resolution of $1.1 \mathrm{~cm}$, the $20 \mathrm{~cm}$ cycle may do attribute to the seasonal variability of precipitation. There's a possibility that the common $20 \mathrm{~cm}$ cycle is just an artificial identification by chance. Parts of the paper should be rearranged. For instance, it's very strange for Fig. 10, together with a short discussion on the distribution of isotopic composition, to be firstly appeared in Conclusions.

Interactive comment on The Cryosphere Discuss., doi:10.5194/tc-2016-263, 2016. 